\title{
CHARACTERIZATION OF A NOVOLAC RESIN SUBSTITUTING PHENOL BY AMMONIUM LIGNOSULFONATE AS FILLER OR EXTENDER
}

\author{
Juan Manuel Pérez ${ }^{\mathrm{a}}$, Francisco Rodríguez ${ }^{\mathrm{a}}$, M. Virginia Alonso ${ }^{\mathrm{a}}$, Mercedes Oliet $^{\mathrm{a},{ }^{*}}$, and \\ Juan M. Echeverría ${ }^{b}$ \\ In this work two types of lignin-novolac resins have been formulated, \\ partially substituting phenol by softwood ammonium lignosulfonate as \\ filler or extender (methylolated) to study the viability of that substitution \\ when resins will be employed as adhesives in textile felts. A commercial \\ novolac resin was used as reference. Free phenol, free formaldehyde, \\ water content, softening point, and flow distance values were determined \\ in all cases to verify whether the material fulfills specifications. In \\ addition, FTIR and NMR spectroscopic techniques were employed for \\ the characterization of three resins samples tested to discuss their \\ structural differences and similarities. The results obtained have shown \\ that the substitution proposed is feasible from the point of view of the \\ resins synthesis to get the pre-polymer.
}

Keywords: Phenolic Resins, Ammonium Lignosulfonate, Methylolation, Synthesis

Contact information: a: Departamento de Ingeniería Química, Facultad de Ciencias Químicas, Universidad Complutense de Madrid, Avda. Complutense s/n. 28040 Madrid. Spain; b: Hexion Speciality Chemicals Ibérica S.A.,Ctra. a Navarra, Epele 39, 20120 Hernani, Spain; *Corresponding author: moliet@quim.ucm.es

\section{INTRODUCTION}

There are two types of phenolic resins: resol and novolac. The first one is synthesized under basic $\mathrm{pH}$ conditions with excess of formaldehyde, and the latter is carried out at acidic $\mathrm{pH}$ (with an excess of phenol). They are widely used in industry because of their chemical resistance, electrical insulation, and dimensional stability (Gardziella et al. 2000). There are some published works involving lignin-resol resins (Kou et al. 1991; Vázquez et al. 1997; Danielson et al. 1998; Alonso et al. 2001), fewer employing lignin-novolac for different applications (El-Saied et al. 1984; Ysbrandy et al. 1992), but there has not been any work involving lignin-novolac combinations for textilefelts applications, which is the focus of the present paper. Phenolic resin-bonded textile felts can be considered to be fiber-reinforced plastic with a high level of fibers. These fibers are derived from textile scraps recycled from the textile industry.

Due to the increase of phenol cost, researchers have been working to partially substitute this monomer by natural polymers that present similar structure in the resin without modification of resin properties (Gardziella et al. 2000). One of these possible substitutes, among other natural compounds, is lignin, a polydisperse natural polymer constituted mainly of phenyl propane units, which present a structure close to that of phenolic resin (Forss et al. 1979). 
Lignin is a by-product of the pulp and paper industry that can be present in many different forms. The choice of the type of lignin to incorporate into resin synthesis has been based on cost and availability (Alonso et al. 2001). The kraft process consumes most of the lignin in the process of recovering the reagents used in pulping. Organosolv lignins are not available in great amounts. So, the best possible substitutes of phenol by lignin-derived chemicals at an industrial scale are the lignosulfonates, which are obtained from the sulphite process. Among the different types of lignosulfonate, the ammonium lignosulfonate is the most suitable to substitute phenol, because final properties of phenolic resins are better (Calvé et al. 1988; Allan et al. 1989). In a previous work it has been verified that the lignosulfonate that better adapts as copolymer in the formulation of resol type phenolic resins was the ammonic type from coniferous wood (Alonso et al. 2001). The results of that study make it possible to deduce that this type of lignin also can be useful for novolac resins. In addition, ammonium ion is used as an intermediate in the first stage of the curing reaction of the formulated resin, which favors this process (Zhang et al. 1997).

Lignosulfonates are not very reactive with phenol and formaldehyde; hence it is also usual to modify their structure. The most common ways of lignin modification are methylolation (Dolenko et al. 1978; Peng et al. 1994; Alonso et al. 2001) and phenolation (Nada et al. 1987; Alonso et al. 2005). Both processes are able to activate lignin, but phenolation is less studied because of the high cost of this process. On the other hand, methylolation does not need an expensive installation.

Novolac oligomers are prepared in acidic media, using an excess of phenol over formaldehyde. The mechanism associated with this reaction can be described in four steps. First, a methylene glycol is protonated by an acid from the reaction medium, which then releases water to form a hydroxymethylene carbonium ion. This ion acts as a hydroxyalkylating agent by reacting with phenol via electrophilic aromatic substitution. A pair of electrons from the benzene ring attacks the electrophile, forming a carbocation intermediate, followed by deprotonation and a regain of aromaticity. The methylol group of the hydroxymethylated phenol is unstable under acidic conditions and loses water readily to form a benzylic carbonium ion. This ion then reacts with another phenol to form a methylene bridge in another electrophilic aromatic substitution in the ortho and para positions. This major process repeats itself until the formaldehyde is exhausted (Knop and Pilato 1985). These pre-polymers are thermally stable and can be stored effectively. Novolac crosslinking is usually achieved by introducing a source of methylene groups to form additional methylene bridges between aromatic rings. Hexamethylenetetramine (HMTA) is the most widely used curing agent (source of formaldehyde) for these reactions.

The reaction mechanism associated with the formulation of lignin-novolac is similar to that of the conventional approaches previously described. Considering the particular case of the lignosulfonates employed in this work, the $\alpha$-carbon of the lateral chain of the phenyl-propane units is occupied by the sulfonate group. Lignosulfonates may be reacting by itself or with phenol adding to the $\beta$-carbon of the lateral chain. The proposed mechanism for the reaction among the lignosulfonate with phenol and formaldehyde in acid medium is exhibited in Fig. 1 (Dos Santos, 1996). The first step consists of the condensation between lignin fragments and the phenol present with 
formaldehyde. After that, in a second step, vacuum distillation is employed to adjust the content of free phenol in accordance with the required specifications for textile felt applications. The addition of a curing agent (HMTA) with methylene groups is also necessary for the crosslinking.

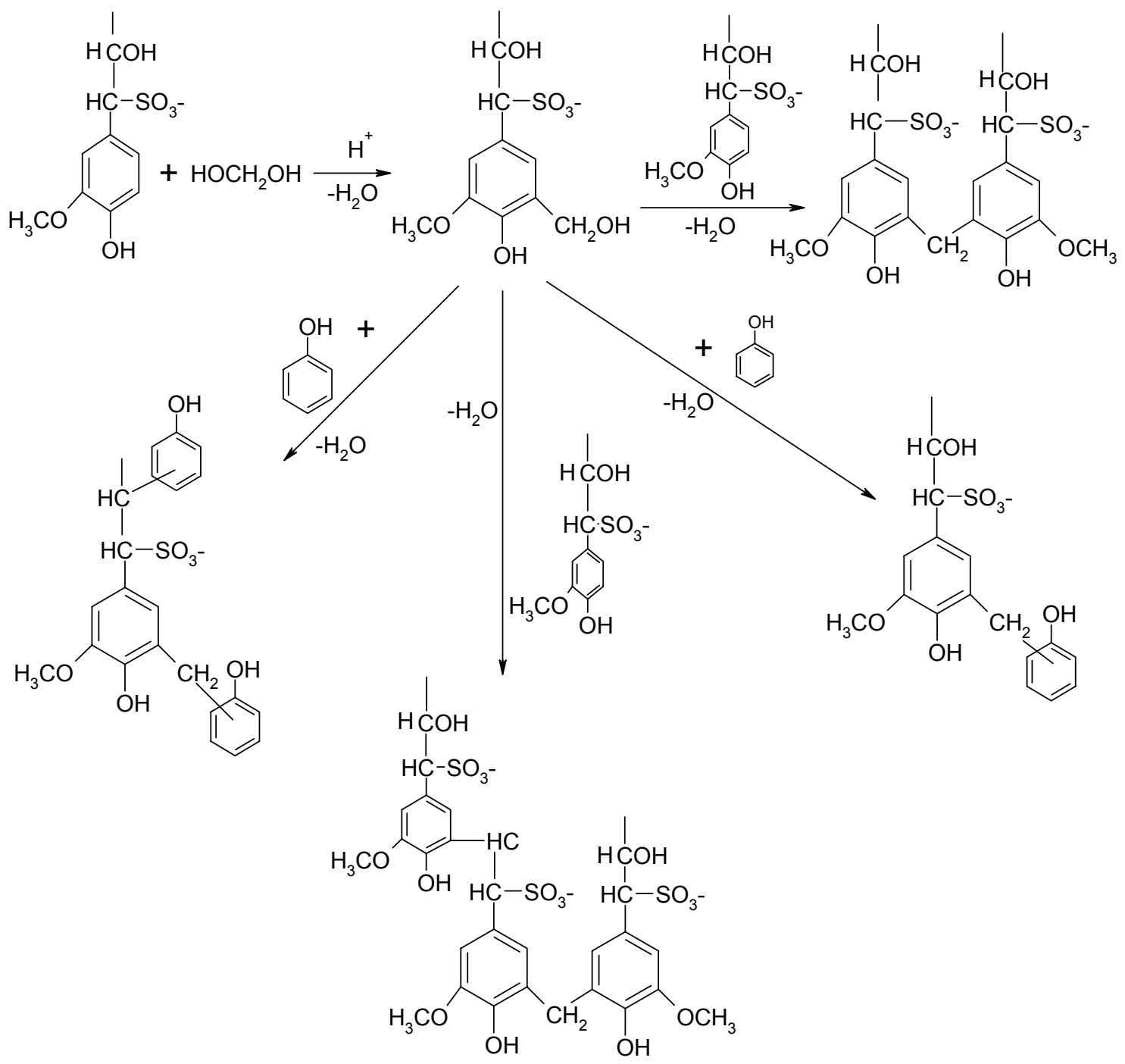

Figure 1. Mechanism of lignin-novolac resins synthesis substituted with lignosulfonates

In this work, two types of lignin-novolac resins were synthesized in the laboratory and compared with a commercial novolac. One lignin-novolac resin was formulated incorporating softwood ammonium lignosulfonate directly, as filler, and the other one incorporated ammonium lignosulfonate modified by methylolation. Formulated resins were characterized in terms of free phenol, free formaldehyde and water contents, softening point, and flow distance to compare with properties of commercial resin. The structural differences among resins were established spectroscopically by Fourier Transform Infra-Red (FTIR) spectroscopy and ${ }^{1} \mathrm{H}$ and ${ }^{13} \mathrm{C}$ Nuclear Magnetic Resonance 
(NMR) techniques. The aim of this work was to study whether the partial substitution of phenol by lignin is feasible, attending to their chemical structure and the specifications for the final applications.

\section{EXPERIMENTAL}

\section{Materials}

Commercial novolac resin and hexamethylenetetramine were supplied by Hexion Speciality Chemicals Ibérica, S.A. The softwood ammonium lignosulfonate used was supplied by Borregaard Deutschland as Borresperse AM 320. This product resembles a spray-dried powder containing $80 \mathrm{wt} \%$ of lignosulfonate, $6 \%$ of ash, $4.22 \%$ of moisture, and $1.93 \%$ of phenolic hydroxyl groups. Lignin-novolac resins were synthesized by using commercial grades of phenol, formaldehyde, and oxalic acid.

\section{Procedure}

A commercial novolac resin (PF) was tested in this study as reference material. This product is obtained by the polymerization between formaldehyde and phenol in acidic medium. Lignin-novolac (LN) was formulated in the laboratory, substituting phenol by lignosulfonate at the beginning of the pre-polymer synthesis (Fig. 1). Methylolated lignin-novolac (MLN) resin was synthesized under the same operating conditions as LN resin, but using lignosulfonate modified by methylolation. The details of this modification were reported in a previous article (Alonso et al. 2001). The substitution of phenol by ammonium lignosulfonate has been $30 \mathrm{wt} \%$ in both samples, because of higher amounts do not produce suitable resins (Ysbrandy et al. 1992; Kharade et al. 1998). The phenol/lignosulfonate (P/L) and phenol- lignosulfonate/formaldehyde $(\mathrm{PL} / \mathrm{F})$ molar ratios were $1 / 0.27$ and $1 / 0.76$, respectively.

The resins syntheses (LN and MLN) were carried out in a laboratory glass reactor (2 1) equipped with a stirrer, thermometer, and reflux condenser (Fig. 2.a), and were accomplished in four stages. First was the addition step, where lignosulfonate (modified or non-modified), phenol, and oxalic acid ( $0.5 \mathrm{wt} \%$ in relation to phenol) were dissolved and heated up to a temperature of $100{ }^{\circ} \mathrm{C}$. Then, the formaldehyde $(37 \mathrm{wt} \%)$ was added, with heating continued during $90 \mathrm{~min}$. Second, the condensation reaction took place for 90 min (Fig. 2.a.). After the second stage, the installation was changed by a distillation system, as shown in Fig. 2.b. The water was removed by atmospheric pressure distillation followed by vacuum distillation to remove most of phenol and water. Finally, the resin was washed with distillated water to remove the phenol and achieve the required specifications.

\section{Pre-Polymers Analysis}

The parameters analyzed in the formulated samples were free phenol, free formaldehyde, water content, softening point, and flow distance. The values for these parameters must be in agreement with commercial resin specifications. 
a)

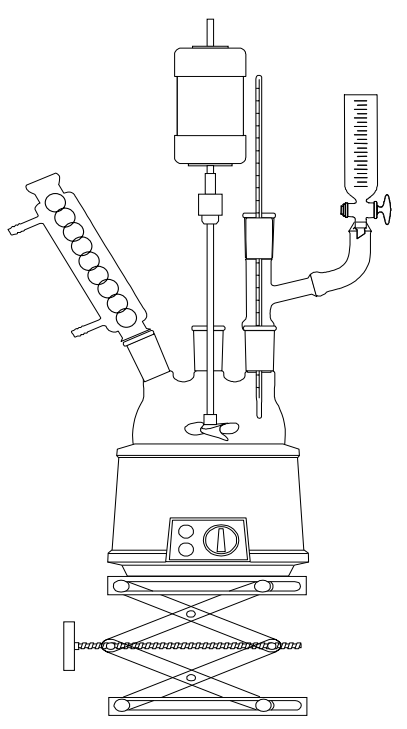

b)

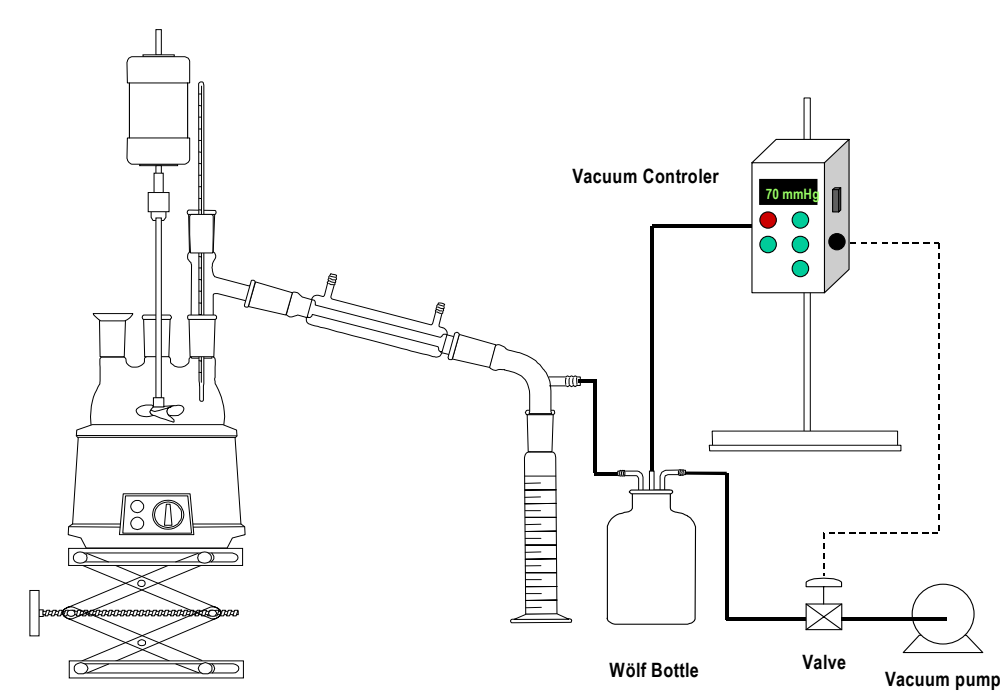

Figure 2. Scheme of experimental set-up of resins synthesis. a) Formaldehyde addition and reaction condensation; b) Atmospheric and vacuum distillations

Free phenol $\left(P_{f}\right)$ was determined by gas chromatography, using a Varian 3400 chromatograph with a flame ionization detector (FID) and helium as carrier gas. An HPINNOWax (crosslinked polyethylene glycol) high-performance capillary column of 0.2 $\mu \mathrm{m}$ film thickness, $50 \mathrm{~m}$ length, and $0.2 \mathrm{~mm}$ internal diameter was used. The internal standard employed was $p$-cresol, which was added to the methanol dissolved samples.

Free formaldehyde $\left(F_{f}\right)$ was analyzed by the hydroxylamine hydrochloride method with endpoint titration (ISO 9397) by using a METTLER TOLEDO titrator DL-50. Free formaldehyde was also analyzed in the methylolated lignosulfonate in order to determinate its conversion in the reaction and formulation of prepolymer.

The resin or methylolated lignosulfonate $(1 \mathrm{~g})$ was dissolved in $60 \mathrm{~mL}$ of an isopropanol-water mixture $(2: 1, \mathrm{v} / \mathrm{v})$; hydrochloric acid was added until the $\mathrm{pH}$ was between 2 and 3. Then, $0.1 \mathrm{~N}$ sodium hydroxide was used to adjust the $\mathrm{pH}$ to 3.5. Finally, $10 \mathrm{~mL}$ of the $10 \%$ hydroxylamine-hydrochloride solution was added. After $10 \mathrm{~min}$, the solution was retitrated with $0.1 \mathrm{~N}$ sodium hydroxide up to $\mathrm{pH}=3.5$.

Water content $\left(W_{f}\right)$ was determined according to DIN 51777.1 and DIN 53715 by using a Karl-Fischer Titrator (DL-31 Mettler-Toledo).

Softening point $(S P)$ was performed in a Mettler-Toledo FP-900 with a FP83 measurement cell according to DIN 51920.

Flow distance $(F D)$. From the powdered resin (mixture of sample novolac resin and $11 \mathrm{wt} \%$ HMTA), a tablet was formed. This tablet was placed on a pre-heated horizontal glass plate at $125^{\circ} \mathrm{C}$ in an oven (ISO 8619). After $3 \mathrm{~min}$, the plate was tilted to an angle of $60^{\circ}$. The flow distance was measured after $30 \mathrm{~min}$ and given in $\mathrm{mm}$. 


\section{FTIR/NMR Spectroscopy}

The commercial novolac, lignin-novolac, and methylolated lignin-novolac resins were characterized by FTIR and ${ }^{1} \mathrm{H}$ and ${ }^{13} \mathrm{C}$ NMR spectroscopic techniques in order to analyze the structural differences. In addition, the lignosulfonates (modified and without modifying) employed were also analyzed by using these techniques.

FTIR spectra were recorded with a Mattson Satellite spectrophotometer, using the potassium bromide pellet method. The pellet was prepared from a mixture of $300 \mathrm{mg}$ potassium bromide and $5 \mathrm{mg}$ of resin sample (or lignosulfonate). The operating conditions were: $4000-400 \mathrm{~cm}^{-1}$ spectral width, 32 accumulations, 1 gain, $4 \mathrm{~cm}^{-1}$ resolution, and signal processing by triangular apodization. Band asignments were based on cited studies (Morterra et al. 1985; Costa et al. 1997; Nada et al. 1998; Carotenuto et al. 1999; Sun et al. 2001).

${ }^{1} \mathrm{H}$ and ${ }^{13} \mathrm{C}$ NMR spectroscopy. The ${ }^{1} \mathrm{H}$ spectra were recorded on a $200 \mathrm{MHz}$ NMR spectrometer (BRUKER AC 200). The conditions used were: $4000.000 \mathrm{~Hz}$ sweep width, $7.2 \mu \mathrm{s}$ pulse width, and a temperature of $297 \mathrm{~K}$. Samples $(0.1 \mathrm{~g})$ and $10 \mu \mathrm{L}$ of tetramethylsilane (TMS) were dissolved in $1 \mathrm{ml}$ of deuterated-dimethylsulfoxide $\left(\mathrm{DMSO}_{\mathrm{d} 6}\right) .{ }^{1} \mathrm{H}$ chemical shifts were measured with respect to TMS as internal standard $\left\{\left(\mathrm{DMSO}_{\mathrm{d} 6}\right)=2.5 \mathrm{ppm}\right\} .{ }^{13} \mathrm{C}$ spectra were recorded on a $500 \mathrm{MHz}$ NMR spectrometer (BRUKER AMX 500). In this case, the conditions used were: $90^{\circ}$ pulse width, $6 \mathrm{~s}$ pulse time, $323 \mathrm{~K}$, and 26000 sweeps. Peaks assessments were based on cited literature (Lundquist et al. 1989; Faix et al. 1994; Gardziella et al. 2000; Sun et al. 2001; Wang et al. 2005).

\section{RESULTS AND DISCUSSION}

\section{Characterization of Novolacs}

The required specifications for commercial (PF) resins and the results obtained in the characterization of lignin-novolac (LN) and methylolated lignin-novolac (MLN) are shown in Table 1. The free formaldehyde amounts of different samples fulfilled the specifications because these polymers are formulated with formaldehyde by default. In relation to free phenol content, lignin-resin (LN), and methylolated lignin resin (MLN) also conformed to the required values according to commercial resins specifications. Note that the methylolated lignin-novolac showed a free phenol content superior to that of the lignin-novolac resin. Methylolated lignin resin reduces the permeability in relation to $\mathrm{LN}$ and commercial resins, and therefore the pre-polymer offers resistance to the removal of phenol during the formulation.

Water content is also an important specification in the final application of these resins because it has a remarkable influence on the plasticity of novolacs. The effect on the melt viscosity is even more pronounced. For example, a buildup of the water content from 0.1 to $3 \%$ reduces the melt viscosity up to $90 \%$ (Knop and Pilato 1985). The reactivity of novolac to HMTA increases when water content increases, and the flow distance is reduced in spite of decreasing melt viscosity (Gardziella et al. 2000). The influence of free phenol is similar to water content but is less severe. 
Table 1. Lignin-Resin Characteristics and Commercial Resin Specifications.

\begin{tabular}{lccccc}
\hline Resin & $\mathrm{P}_{\mathrm{f}}(\%)$ & $\mathrm{F}_{\mathrm{f}}(\%)$ & $\mathrm{W}_{\mathrm{f}}(\%)$ & $\mathrm{SP}\left({ }^{\circ} \mathrm{C}\right)$ & $\mathrm{FD}(\mathrm{mm})$ \\
\hline LN & 0.14 & 0.19 & 0.23 & 119 & 27 \\
MLN & 0.19 & 0.02 & 0.28 & 118 & 26 \\
Commercial $^{*}$ & $<0.20$ & $<0.30$ & $<0.40$ & $115-125$ & $25-40$ \\
\hline
\end{tabular}

$P_{f}$ : Free phenol; $F_{f}:$ Free formaldehyde; $W_{f}:$ Water content; SP: Softening point; FD: Flow distance. * Required specifications supplied by Hexion Ibérica, S.A.

In addition, curing reaction of novolacs is carried out over $100{ }^{\circ} \mathrm{C}$, water forms vapor, and so the final material can present a porous structure with reduced mechanical properties. Therefore, a resin with low initial water content will achieve better final properties after curing. Note that all resins studied had less water than the maximum commercial specification, and lignin-novolac had less water than MLN because less water was used during formulation of LN resin (Table 1).

The softening point is a critical parameter in order to determine the feasibility of resins in textile-felt applications. If the softening point of lignin-resins is not in the commercial resin range $\left(115-125{ }^{\circ} \mathrm{C}\right)$, then these pre-polymers would not be employed for textile-felts, because the resin could not penetrate into the fibers. In this case, the softening point temperatures of synthesized resins with ammonium lignosulfonate were in the specified range, as shown in Table 1.

Lignin-novolac and methylolated lignin-novolac resins had a flow distance within the commercial range, but these results were near the lower limit. This fact is due to the presence of lignin in the samples, which reduced chain mobility of the resins. In short, these two lignin-resins fulfilled the specifications required by the textile-felts manufacture.

The FTIR spectra of lignin-novolac, methylolated lignin-novolac, and commercial resins are shown in Fig. 3. The highest wavenumber zone did not offer any difference among the three resins assayed. However, the spectra showed more information about resins structure within the 2000 to $400 \mathrm{~cm}^{-1}$ zone. The broad absorbance at 1701-1692 $\mathrm{cm}^{-1}$ corresponding to $\mathrm{C}=\mathrm{O}$ stretching was observed in MLN spectra, but not in the commercial spectrum (Faix et al. 1994; Nada et al. 1998). The most typical band of lignin structures is $1600 \mathrm{~cm}^{-1}$. In this case, it was not present in commercial resin, but a 1610 $\mathrm{cm}^{-1}$ band was able to overlap it. In addition, the $1470-1460 \mathrm{~cm}^{-1}$ band was not very intensive, which indicates that methylolated lignin molecule incorporates hydroxylmethyl groups in the MLN resin (Nada et al. 1998; Alonso et al. 2001). The main objective of methylolation was to incorporate hydroxylmethyl groups into the lignin aromatic ring. Note that LN resin also showed the same band $\left(1470-1460 \mathrm{~cm}^{-1}\right)$, which implies that even without methylolation this group can be incorporated into the aromatic ring of lignin. In addition, this band (1470-1460 $\mathrm{cm}^{-1}$ ) overlapped with a $1430 \mathrm{~cm}^{-1}$ band (methylene bridge), which is typical of novolac resins and in this case is not well resolved (Costa et al. 1997). The $819 \mathrm{~cm}^{-1}$ band corresponds to out-of-plane aromatic ring deformation by 1 , 2 , and 4 links. The band at $754 \mathrm{~cm}^{-1}$ is the 1,2 , and 6 substitutions of this aromatic ring. These two bands are quite similar in all resins due to great similarity of the substitutions, 
as shown in Fig. 3. On the other hand, lignin-novolac and methylolated lignin-novolac had a predominance of 1, 2, and 6 links. This means that lignin aromatic rings have steric hindrance (Ŝebenik et al. 1974; Costa et al. 1997). The $655 \mathrm{~cm}^{-1}$ band in LN and MLN resins spectra indicates the presence of sulfonic groups from lignosulfonate.

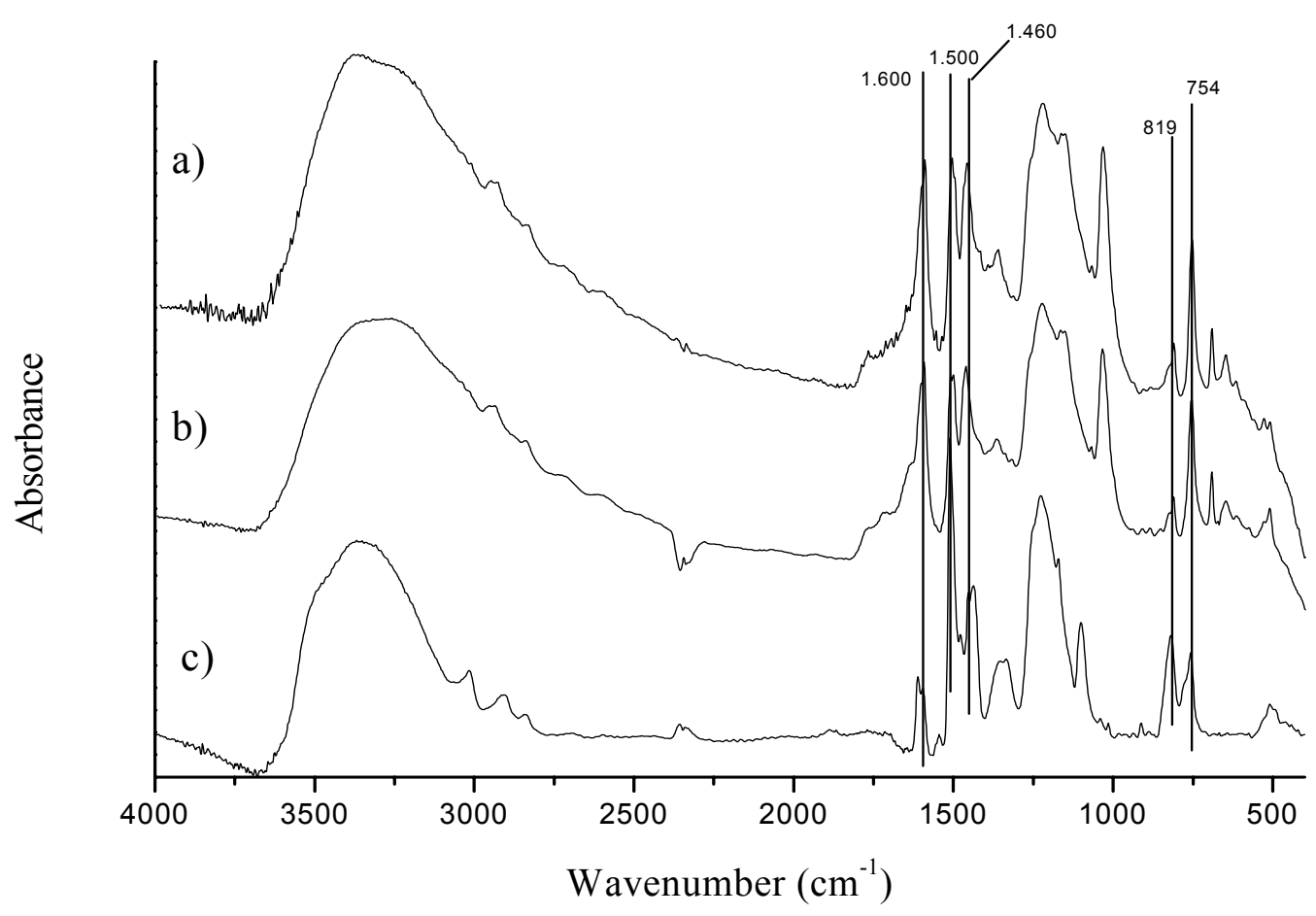

Figure 3. FTIR spectra of a) $L N$, b) MLN and c) Commercial novolac resins

The three resins studied also showed important differences in ${ }^{1} \mathrm{H}$ NMR spectra (Fig. 4). For instance, the $9 \mathrm{ppm}$ zone corresponds to aldehyde protons linked to aromatic ring substitutions of the type -CHO. This signal did not appear in MLN, which implies that in this pre-polymer there were higher levels of substitutions than in the others resins. In addition, it supposes more steric hindrance to - $\mathrm{CHO}$ group incorporation into the MLN aromatic rings. Signals corresponding to aromatic protons $(6.5-7.0 \mathrm{ppm})$ showed the presence of more free positions in the aromatic rings of resins. An absence of signals in the range of 7.0 to $7.5 \mathrm{ppm}$ for commercial resin spectrum showed that the aromatic rings were less substituted. The lignin-novolac and methylolated lignin-novolac resins exhibited a higher level of replacement at the aromatic ring, which indicates that crosslinking reactions will be favored during the curing stage. In lignin-novolac resins the decrease of protons signal of hydroxylmethyl groups coming from lignin (4.5-4.6 ppm) demonstrated the dehydration of these groups to form methylene links with phenyl groups; this dehydration did not happen in commercial resins, which explain higher reactivity of lignin-novolac with the phenol. Thus, these circumstances are desirable for 
resin-curing reactions. 3.80-3.90 ppm signals appeared in the MLN resin spectrum, which indicates the presence of $-\mathrm{CH}_{2} \mathrm{OH}$ groups coming from the methylolation reaction. This group could promote greater crosslinking of MLN resin during the curing reaction.

The resins ${ }^{1} \mathrm{H}$ NMR spectra did not reveal the complexity of the chemical structure of these polymers, due to the overlapping of signals (Fig. 4). The ${ }^{13} \mathrm{C}$ NMR technique, in general, allows identification of a greater number of functional groups. Lignin-novolac spectra have a great many signals due to presence of lignin functional groups, which have a very complex structure. ${ }^{13} \mathrm{C}$ NMR spectra give an idea of the difficulty in understanding the resins structure, as shown in Fig. 5.

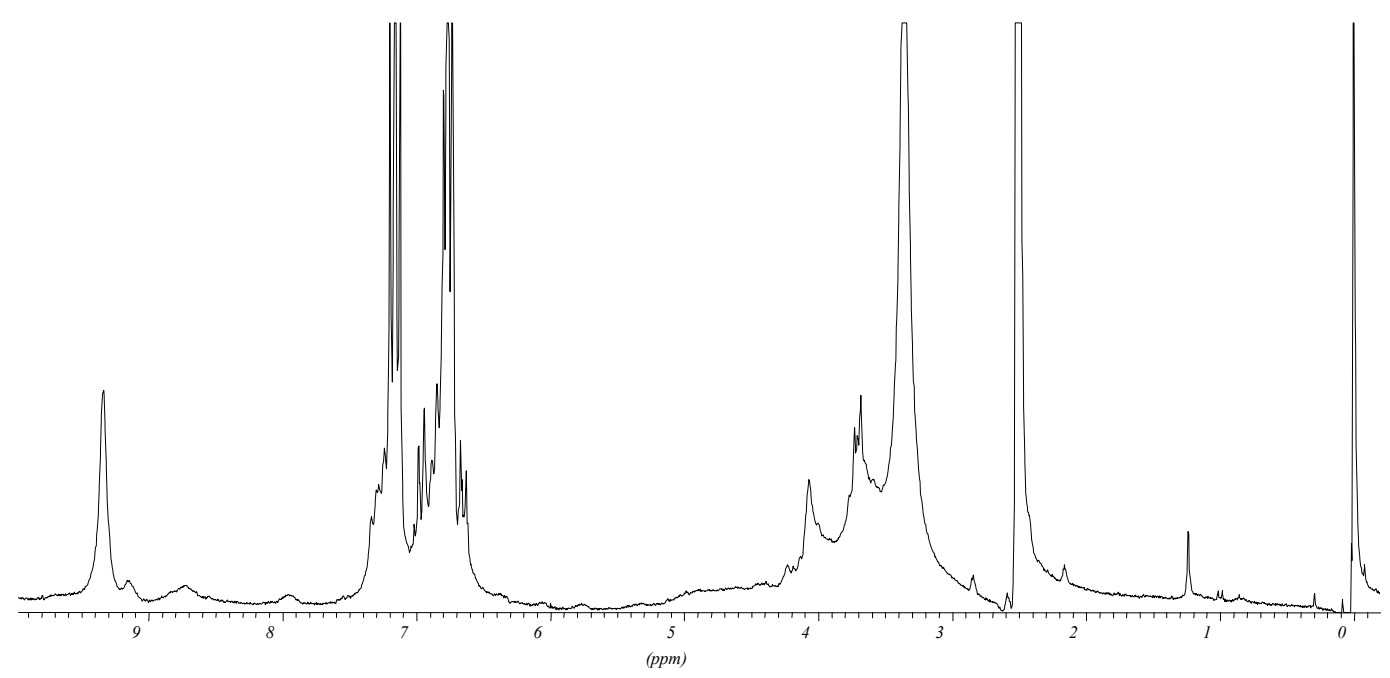

Figure 4 a. ${ }^{1} \mathrm{H}$ NMR spectrum of $L N$ resin

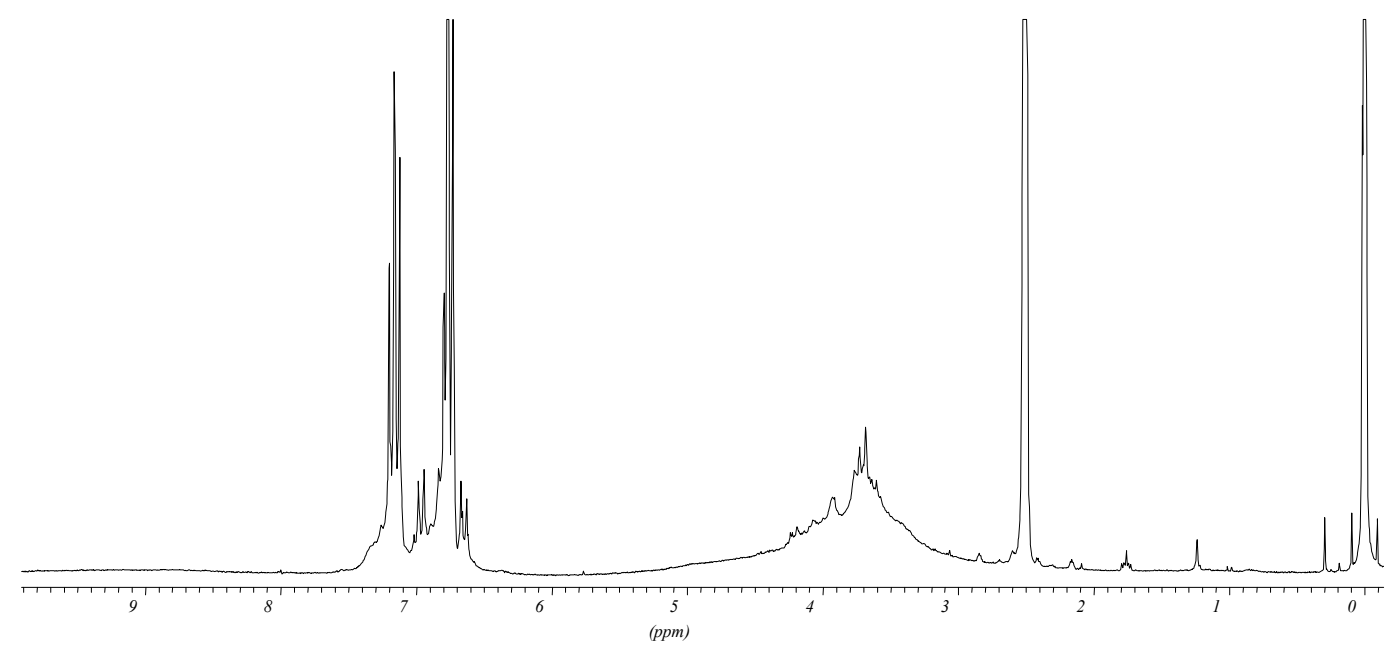

Figure 4 b. ${ }^{1} \mathrm{H}$ NMR spectrum of MLN resin 


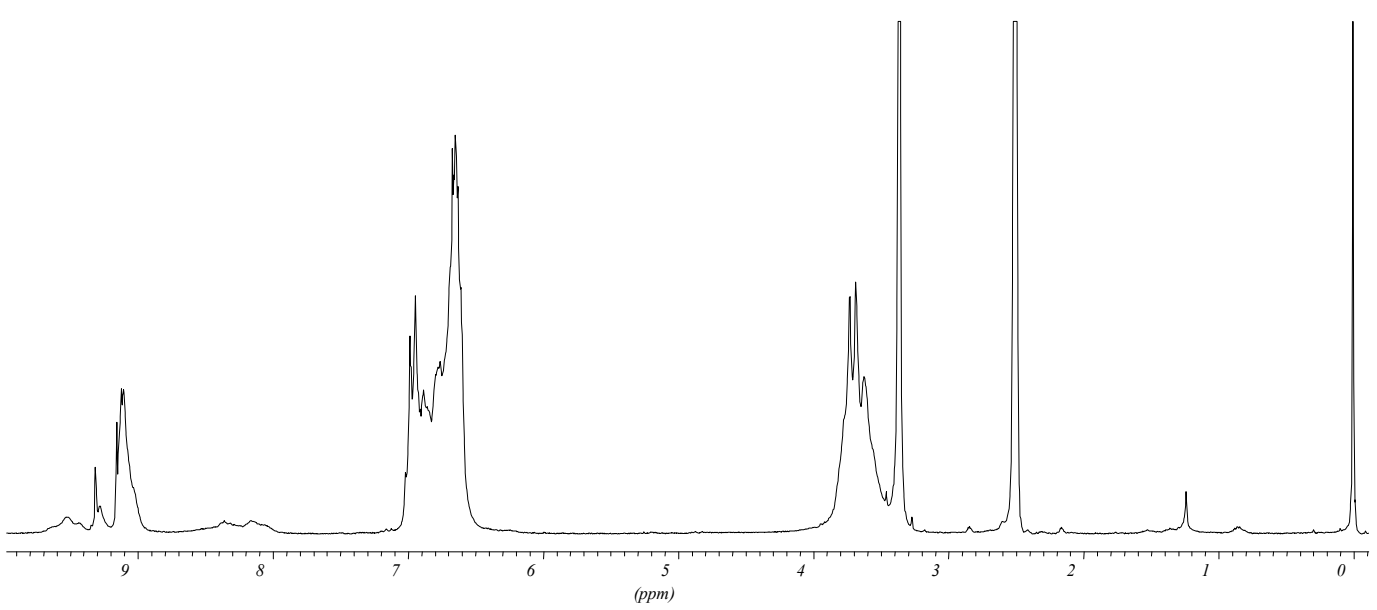

Figure 4 c. ${ }^{1} \mathrm{H}$ NMR spectrum of commercial novolac resins

An absence of peaks at 170-180 ppm implies that the aliphatic carboxylic acids were not formed in any of the three resins studied, which could make the resin curing reactions more difficult. The $166.5 \mathrm{ppm}$ peak, which appears in the MLN spectrum, corresponds to the $\mathrm{C}=\mathrm{O}$ link. This feature was also observed in the FTIR spectrum (Fig. 3.b), and it can be assigned to ester group linked to aromatic ring. The spectrum of commercial resin showed a signal at $150 \mathrm{ppm}$ corresponding to $\mathrm{OH}$ linked to aromatic carbon, and this signal was unsubstituted in the ortho position by a $\mathrm{CH}_{2}$ in $\mathrm{LN}$ and MLN. The same happens around $130 \mathrm{ppm}$, which is also a typical signal of novolac resins, due to $\mathrm{C} 3$ and $\mathrm{C} 5$ carbons of the phenolic ring substituted in its ortho position by a $\mathrm{CH}_{2}$ (Holopainen et al. 1997). This signal, in novolac resins, is masked by resonances coming from lignosulfonate incorporated during polymer synthesis. The zone of $60-80 \mathrm{ppm}(\alpha, \beta$ and $\gamma$ carbons of the ( $\beta$ )- aliphatic-O-(4)-aromatic links) was present only in the ligninsubstituted resins. This range is consistent with the presence of a lignin structure. The zone of $35 \mathrm{ppm}$, characteristic of phenolic resins, is due to $\mathrm{CH}_{2}$ in an ortho position with respect to the hydroxylmethyl carbon group of phenolic ring (Holopainen et al. 1997).

Note that the incorporation of methylolated lignin instead of lignosulfonate presents a reaction mechanism similar to that shown in Fig. 2, with the exception that the formaldehyde cannot occupy meta sites in an aromatic ring. Although both mechanisms are similar in lignin samples, methylolated lignin presents methylene groups which will react with the phenol, while lignosulfonate (without modification) competes with methylene links between phenolic rings.

\section{Summary of Results}

Summarizing, despite a great number of signals and unavoidable overlapping, it was possible to distinguish novolac's typical signals by means of the three techniques employed. Identification of these signals implies that the lignin-novolac and methylolated lignin-novolac resins had similar links relative to commercial resin; however, resins synthesized with lignin showed a more complicated structure, due to lignin incorporation. Thus, LN and MLN would substitute for commercial resins in textile felt applications. 
In the future an extensive characterization would be needed to determine and optimize cure process conditions for these polymers. In addition, other tasks should include evaluation of curing agent content (HMTA) during the cure process of the resins. Processing knowledge and kinetic methods developed for epoxy and unsaturated polyester resins should also benefit the manufacture of similar systems based on different polymers (Kenny et al. 1995; Boey et al. 2000; Lee et al. 2000).

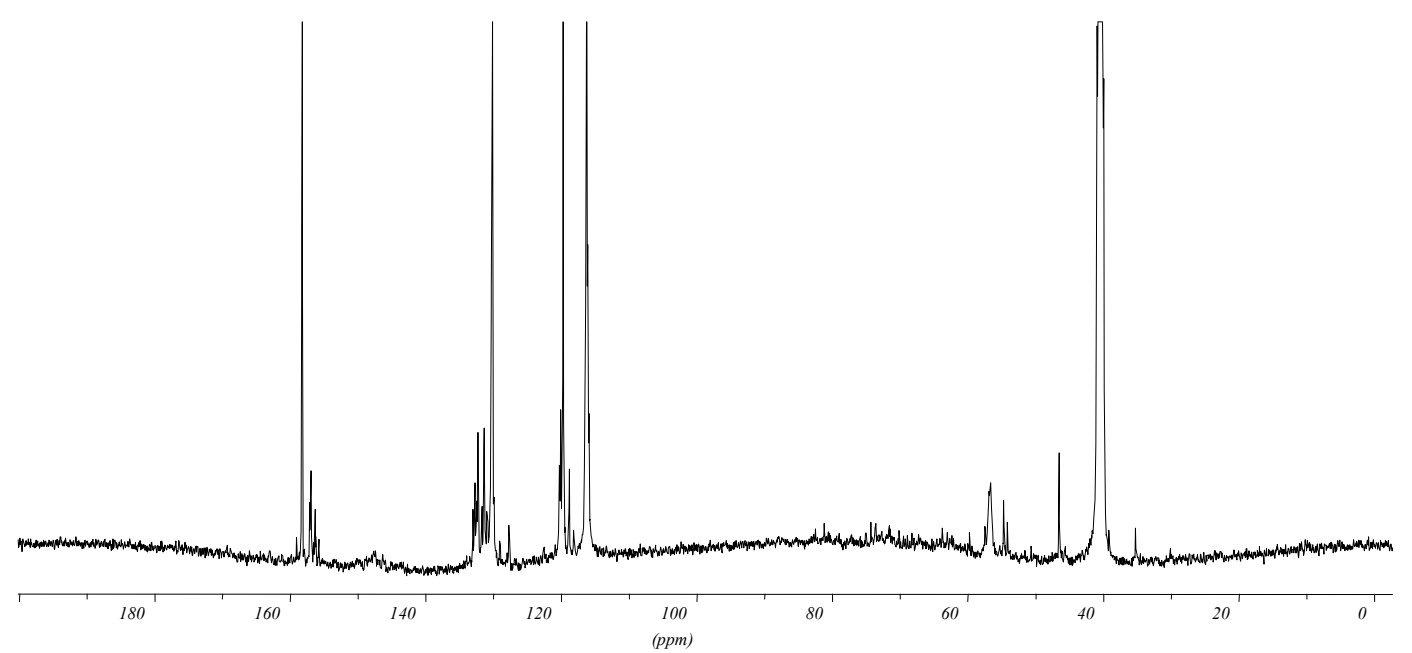

Figure 5 a. ${ }^{13} \mathrm{C}$ NMR spectrum of $L N$ resin

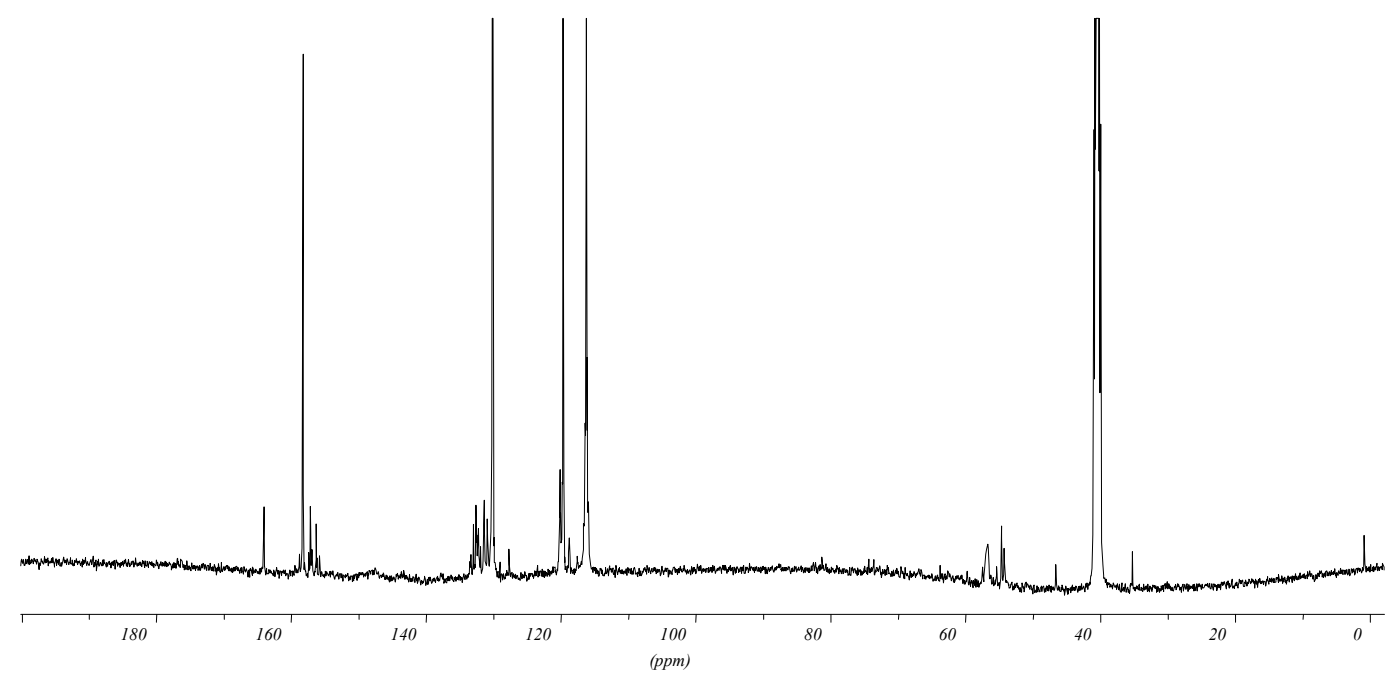

Figure 5 b. ${ }^{13} \mathrm{C}$ NMR spectra of MLN resin 


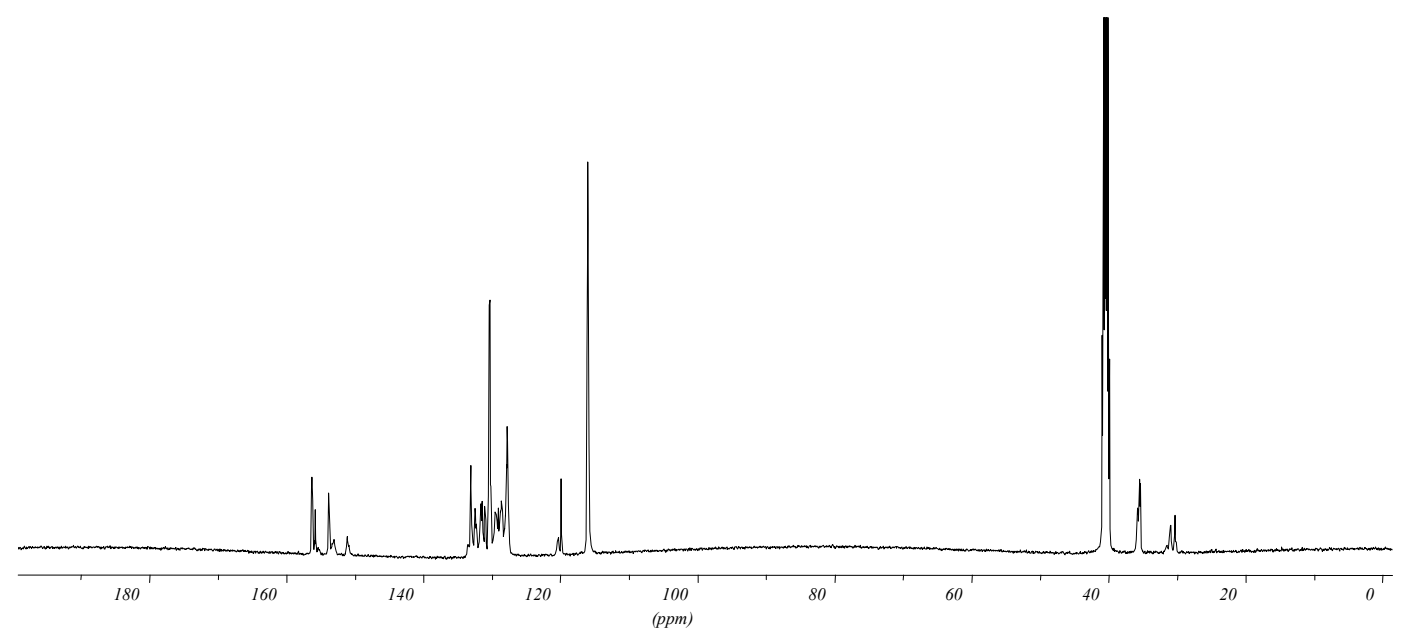

Figure 5 c. ${ }^{13} \mathrm{C}$ NMR spectra of commercial novolac resin

\section{CONCLUSIONS}

1. Phenol, formaldehyde, and water contents, as well as softening point and flow distance values of LN and MLN resins synthesized were in range with specifications that have been established for use of commercial novolac resins. Therefore, these samples conform to the specifications required for the textile felt applications.

2. FTIR and NMR spectroscopies showed that the chemical structure of lignin-novolac and methylolated lignin-novolac resins were more complicated than that of a commercial novolac resin due to the functional groups of the incorporated ammonium lignosulfonate.

3. Although the LN and MLN resins are structurally complex, the high variety of functional groups in relation to commercial resin could favour the curing reaction.

\section{ACKNOWLEDGEMENTS}

The authors gratefully acknowledge the support of "Ministerio de Ciencia y Tecnología" (project CTQ2004-02031/PPQ).

\section{REFERENCES CITED}

Alonso, M. V., Rodríguez, J. J., Oliet, M., Rodríguez, F., García, J., and Gilarranz, M. A. (2001). "Characterization and structural modification of ammonic lignosulfonate by methylolation," J. Appl. Polym. Sci. 82(11), 2661-2668.

Alonso, M. V., Oliet, M., Rodríguez, F., García, J., Gilarranz, M. A., and Rodríguez, J. J. (2005). "Modification of ammonium lignosulfonate by phenolation for use in phenolic resins," Bioresource Technol. 96(9), 1013-1018. 
Allan, G. G., Dalan, J. A., and Foster, N. C. (1989). "Modification of lignins for use in phenolic resins," J. Am. Chem. Soc. Symp. Ser. 385, 55-57.

Boey, F. C. Y., and Qiang, W. (2000). "Experimantal modeling of the cure kinetics of an epoxy hexaanhydro-4-methylphatalic anhydride (MHHPA) system," Polymer 41, 2081-2094.

Calvé, L. R., Shields, J. A., Blanchette, L., and Fréchet J. M. J. (1988). “A practical lignin-based adhesive for waferboard/OSB," Forest Prod. J. 38(5), 15-20.

Carotenuto, G., and Nicolais, L. (1999). "Kinetic Study of Phenolic Resin Cure by IR Spectroscopy," J. Appl. Polym. Sci. 74(11), 2703-2715.

Chow, S., and Steiner, P. R. (1979). "Comparisons of the Cure of Phenol-Formaldehyde Novolac and Resol Systems by Differential Scanning Calorimetry ," J. Appl. Polym Sci. 23, 1973-1985.

Costa, L., Rossi di Monterela, L., Camino, G., Weil, E. D., and Pearce, E. M. (1997). "Structure-charring relationship in phenol-formaldehyde type resins," Polym. Degrad. Stabil. 56, 23-25.

Danielson, B., and Simonson, R. (1998). "Kraft lignin in phenol formaldehyde resin. Part 1. Partial replacement of phenol by Kraft lignin in phenol formaldehyde adhesives for plywood," J. Adhesion Sci. 12(9), 923-939.

Dolenko, A. J., and Clarke, M. R. (1978). "Resin blinders from Kraft lignin," For. Prod. J. 28(8), 41-46.

Dos Santos, F. (1996). "Utilizaçao de Lignossulfonatos na preparaçao de resinas fenólicas tipo novolaca e pós de moldagem fenólicos”. Universidade de Sao Paulo, Maestria, 1996.

El-Saied, H., Nada, A. M. A., Ibrahem, A. A., and Yousef, M. A. (1984). "Waste liquors from cellulosic industries. III. Lignin from soda-spent liquor as a component in phenol-formaldehyde resin ," Angew. Makromolek. Chem. 122, 169-181.

Faix, O., Argyropoulos, D. S., Robert, D., and Neirink, V. (1994). "Determination of Hydroxyl Groups in Lignins Evaluation of ${ }^{1} \mathrm{H}-,{ }^{13} \mathrm{C}-,{ }^{31} \mathrm{P}-\mathrm{NMR}$, FTIR and Wet Chemical Methods," Holzforschung. 48(5), 387-394.

Forss, K. G., Fuhrmann, A. (1979). "Finnish plywood, particleboard, and fireboard made with a lignin-base adhesive," Forest Prod J. 29(7), 39-43.

Gardziella, A., Pilato, L. A., and Know, A. (2000). Phenolic Resins: Chemistry, Applications, Standardization, Safety and Ecology. New York: Springer

Holopainen, T., Alvila, L., Rainio, J., and Pakkanene, T. T. (1997). "PhenolFormaldehyde Resol Resins Studied by ${ }^{13} \mathrm{C}-\mathrm{NMR}$ Spectroscopy, Gel Permeation Chromatography, and Differential Scanning Calorimetry," J. Appl. Polym. Sci. 66, 1183-1193.

Ishida, H., and Rodriguez, Y. (1995). "Curing kinetics of a new benzoxazine-based phenolic resin by differential scanning calorimetry," Polymer. 36(16), 3151-3158.

Kenny, J. M., Pisaniello, G., Farina, F., and Puzziello, S. (1995). "Calorimetric analysis of the polymerisation reaction of a phenolic resin," Thermochim. Acta, 269/270, 201211.

Kharade, A. Y., and Kale, D. D. (1998). "Effect of Lignin on phenolic Novolac Resins and Moulding Powder," Eur. Polym. J. 34(2), 201-205.

Knop, A., Pilato, L. A. (1985). Phenolic Resins. Springer-Verlag, New York. 
Kou, M., Hse, C.-Y., and Huang, D.-H. (1991). "Alkali treated Kraft lignin as a component in flakeboard resins," Holzforschung 45, 47-51.

Lee, S. H., Yoshioka, M., and Shiraishi, N. (2000). "Preparation and properties of phenolated corn bran (CB)/phenol/formaldehyde cocondensed resin," J. Appl. Polym. Sci. 77, 2901-2907.

Lundquist, K., and Stern, K. (1989). "Analysis of lignins by ${ }^{1} \mathrm{H}$ NMR spectroscopy," Nordic Pulp Pap. Res. J. 4(3), 210-213.

Matuana, L.M., Riedl, B., and Barry, A.O. (1993). "Caracterisation cinetique per analyse enthalpique differentielle des resins phenol-formaldehyde a base de lignosulfonates," Eur Polym J. 29(4), 483-490.

Morterra, C., and Low, M. J. D. (1985). "I.R. studies of carbons-VII. The pyrolysis of a phenol-formaldehyde resin," Carbon. 23(5), 525-530.

Nada, A. M. A., El-Saied, H., Ibrahem, A. A., and Yousef, M. A. (1987). "Waste liquors from cellulosic industries. IV. Lignin as component in phenol formaldehyde resol resin," J. Appl. Polym. Sci. 33, 2915-2924.

Nada, A. M. A., El-Sakhawy, M., and Kamel, S. M. (1998). "Infra-red spectroscopy study of lignins," Polym. Degrad. Stabil. 60, 247-251.

Ozawa, T. B. (1965). "A new method of analysing thermogravimetric data," Chem. Soc. Jpn. 38(1), 1881-1886.

Peng, W., and Riedl, B. (1994). "The chermorheology of phenol-formaldehyde thermoset resin and mixtures of the resin with lignin fillers," Polymer 35(6), 1280-1286.

Ŝebenik, A., Vizovisek, I., and Lapange, S. (1974). "Determination of kinetic parameters form the reaction between phenol and formaldehyde by differential scanning calorimetry ," Eur. Polym. J. 10, 273-278.

Sun, R. C., Lu, Q., and Sun, X. F. (2001). "Physico-chemical and thermal characterization of lignins from Caligonum monogoliacum and Tamarix spp," Polym. Degrad. Stabil. 72, 229-238.

Vázquez, G., González, J., Freire, S., and Antorrena, G. (1997). "Effect of chemical modification of lignin on the gluebond performance of lignin-phenolics resins," Biores. Technol. 60, 191-198.

Wang, M., Wei, L., and Zhao, T. (2005). "Cure study of addition-cure-type and condensation-addition-type phenolic resins," Eur. Polym. J. 41, 903-912.

Wolfrum, J., and Ehrenstein, G.W. (1999). "Interdependence Between the Curing Structure, and the Mechanical Properties of Phenolic Resins," J. Appl. Polym. Sci. 74, 3173-3185.

Ysbrandy, R.E., Sanderson, R.D., and Gerischer, G.F.R. (1992). "Adhesives from autohydrolysis bagasse lignin. Part I ," Holzforschung 46(3), 249-252.

Zhang, X., Looney, M. G., and Solomon, D. H. (1997). "The chemistry of novolaca resins: $3 .{ }^{13} \mathrm{C}$ and ${ }^{15} \mathrm{~N}$ n.m.r. studies of curing with hexamethylenetetramine," Polymer $38(23), 5835-5848$.

Article submitted: March 19, 2006; First round of reviewing completed: April 12, 2007; Revised version received May 5, 2007; Corrected version approved: May 23, 2007; Article published May 25, 2007. 